NP36 (continued)

Objective: The objective of this study was to determine if the Action Pack Families child as change agent intervention resulted in changes in the home food and physical activity environment of children over the course of a 3-year intervention.

Description: This study was a group-randomized intervention that included all 10 elementary schools in a county in rural Georgia. Action Pack Families was a child as change agent nutrition and physical activity intervention with school, home, and community based components. The intervention was delivered by trained family nutrition educators in partnership with University of Georgia Extension. The school curriculum was designed to be physically active and experiential. Students were taught strategies for sharing what they learned with their families. In addition to the school-to-home activities that engaged families in intervention activities at home, the family component of the intervention included community events, family fun nights, and communication via Facebook. Community recognition and support of the program was enhanced by participation in local events.

Evaluation: Child height and weight were collected at school by the research team; diet, physical activity, and home environment factors were self-reported by parents using a paper-pencil survey. Anthropometric data were collected at the beginning and end of each school year from 3rd through 5 th grade. Parent survey data were collected at the beginning of each school year as well as at the end of 5th grade. Conclusions and Implications: Previous cross-sectional analyses of baseline data from this study suggested that the home environment does not necessarily influence the dietary behavior of children in the same way across all racial/ethnic groups. An analysis of intervention effects on home environment, dietary, and activity variables showed that the intervention impacted key variables for positive changes in the home environment with the effect varying across racial/ethnic groups. Using children as change agents might be effective in altering some home environment variables that could aid in preventing childhood obesity.

Funding: 2012-68001-19625.

\section{NP37 Wave Ripples for Change (Year 5 of 5): 2-Y Childhood Obesity Prevention Intervention Preliminary Findings and Project Outputs}

Siew Sun Wong, PhD, MS, siewsun.wong@oregonstate.edu, Oregon State University, Ballard Hall 105E, Corvallis, OR 97331; Melinda Manore, PhD, RDN, FACSM;
Megan Patton-Lopez, DPH, RDN, Western Oregon University; John Schuna, PhD; Christopher Scaffidi, PhD; Tonya Johnson, MPH; Yu Meng, MS; Cristian Curiel, BS; Darcie Hill, MPH, RDN, Western Washington University; Jonathon Richter, PhD, Salish Kootenai College; Gretchen Dursch, MA, Oregon State University

Objective: Prevent childhood obesity among high school soccer players (HSSP) ages 14-19 years-old in Oregon.

Description: In June 2017 we concluded the 2-y intervention. This 5-year project will conclude in May 2018. Three key program outputs include: A field-tested, 4-lesson, high school sport nutrition curriculum supplemented with three life-skill team-building workshops, and a 3-D animation computer game for experiential learning. The curriculum is being peer-reviewed now; an enhanced cloudbased system named WavePipe field-tested to manage human studies data collection, monitoring, and automated analyses and report generation; and development of a sports mobile game application prototype concept and wireframe.

Evaluation: Among 388 HSSP who completed the study (51\% attrition rate in 2-y; intervention group $n=278$; comparison group $\mathrm{n}=110$ ), $58 \%$ were females, $38 \%$ were Latino, 38\% participated in school breakfast program and/or national school lunch program, mean age was 15.3 (1.1 years). Compared to the comparison group, the intervention group significantly decreased in added sugar intake by $12 \mathrm{~g} / \mathrm{d}$, but had no changes in fruits and vegetable (FV) and saturated fat consumption. Physical activity (PA) was significantly higher in-soccer season than outof-soccer season for both intervention and comparison groups.

Conclusions and Implications: This is the first study to engage HSSP in a 2 -y obesity prevention program targeting sport nutrition education, healthy eating behaviors for growth, performance and health, emphasizing the importance of daily PA and life-skills experiential learning. Overall, FV intakes were maintained over the 2-y intervention but were below the dietary recommendations, although higher than those typically reported in the literature for youth and youth athletes. The 2-y intervention significantly decreased added sugar intake, by lowering the frequency of selecting cake/cookies and ice cream foods. PA recommendations were met during soccer season, but below recommendations out-of-season. Thus, it is important to engage active youth yearlong, helping them to make daily PA a priority in their lifestyle.

Funding: 2013-67001-2041. 